\title{
Quasar and Supermassive Black Hole Evolution
}

\author{
Scott M. Croom ${ }^{1}$, Stephen Fine ${ }^{1,2}$, and the 2SLAQ Survey Team \\ ${ }^{1}$ Sydney Institute for Astronomy, School of Physics, University of Sydney, NSW 2006, \\ Australia \\ Email: scroom@physics.usyd.edu.au \\ ${ }^{2}$ Department of Physics, University of Durham, South Roiad, Durham DH1 3LE, UK
}

\begin{abstract}
We present results from statistical analysis of the largest quasar surveys currently in existence: the Sloan Digital Sky Survey (SDSS), the 2dF QSO Redshift Survey (2QZ), and the 2dF-SDSS LRG and QSO (2SLAQ) Survey. The quasar luminosity function shows highly significant ( $>99.9 \%$ ) evidence of downsizing: the number density of faint quasars peaks at lower redshift than bright quasars. We measure the distribution of quasar broad-line widths as a function of luminosity, particularly focusing on the $\mathrm{Mg}$ II $\lambda 2799$ line. There is a clear trend, such that more luminous quasars show less scatter in line width than fainter quasars. However, the scatter in line width for any given luminosity (particularly in the brightest objects) is so small that it challenges our current understanding of virial black hole mass estimates.
\end{abstract}

Keywords. black hole physics, line: profiles, surveys, galaxies: active, quasars: general, quasars: emission lines

\section{Introduction}

The realization (e.g., Kormendy \& Richstone 1995) that most massive galaxies contain supermassive black holes (SMBHs) has revolutionized the study of galaxy evolution and AGN. These two, once disparate fields, are now tightly bound together. The only simple explanation of the tight correlation between black hole mass and bulge/spheroid mass (or velocity dispersion; e.g., Tremaine et al. 2002) is that the two co-evolve. Quasars, the most luminous of AGN, were once considered to be intrinsically rare objects, but are now thought to be a short-lived phase in the history of most/all massive galaxies. As a result, understanding the strong evolution of the quasar population has taken on new importance, as has the measurement of black hole masses in quasars.

Major progress has been possible over the last few years due to an array of massive new quasar surveys, using highly multiplexed fiber spectroscopy. The largest of these are the Sloan Digital Sky Survey (SDSS; Schneider et al. 2007), the 2dF QSO Redshift Survey (2QZ; Croom et al. 2004), and the 2dF-SDSS LRG and QSO (2SLAQ; Croom et al. 2009a) survey. The combination of these three provides good coverage of the redshiftluminosity plane, with the exception of low luminosities at $z>3$, a regime currently being targeted by the AAOmega-UKIDSS-SDSS (AUS; Croom et al., in preparation) survey on the Anglo-Australian Telescope. The high quality of the spectra in the latest surveys, coupled with an accurate understanding of their selection functions, makes them an invaluable resource. While not having the size or scale of the optical samples, the other growth area has been X-ray surveys, particularly with the advent of Chandra (e.g., Alexander et al. 2003) and XMM-Newton (e.g., Hasinger et al. 2001). A key result of these has been the observation of "AGN downsizing," with the activity in faint AGN peaking at a lower redshift than that of more luminous AGN (e.g., Ueda et al. 2003; Hasinger 
et al. 2005). This is similar to the downsizing seen in the galaxy population (Cowie et al. 1996), where the most massive galaxies formed the bulk of their stars earlier than lower mass galaxies (e.g., Juneau et al. 2005).

Possibly the central question for the study of quasars is to determine the mass of the supermassive black holes that drive them. This has led to substantial effort, first in reverberation mapping (e.g., Wandel et al. 1999), and then in applying this to the socalled "virial technique" (e.g., McLure \& Jarvis 2002). The virial method takes the broadline radius-luminosity relation derived from reverberation mapping and combines this with the velocity width of the broad lines from single-epoch spectra (see the contributions of Peterson, Vestergaard, and others in these proceedings).

In this paper, we will first present the latest results for the optical luminosity function of quasars, using a combination of the SDSS and 2SLAQ surveys to tightly constrain the bright and faint ends of the quasar luminosity function. We will then study distribution of quasar broad-line widths as a function of luminosity and discuss the implications for black hole mass estimates. Throughout this paper we assume a cosmological model with $H_{0}=70 \mathrm{~km} \mathrm{~s}^{-1} \mathrm{Mpc}^{-1}, \Omega_{\mathrm{m}}=0.3$ and $\Omega_{\Lambda}=0.7$. All photometric measurements quoted in this paper have been corrected for Galactic extinction using the maps of Schlegel, Finkbeiner, \& Davis (1998). Much of the work presented here is based on that of Croom et al. (2009b; henceforth C09b) and Fine et al. (2008).

\section{Quasar Evolution and Downsizing}

\subsection{The 2SLAQ Survey}

The 2SLAQ quasar sample (Croom et al. 2009a; henceforth C09a) is the deepest large area survey in existence. It provides the best available probe of the faint end of the luminosity function of optical quasars. The 2SLAQ survey consists of SDSS selected targets, followed up with spectroscopy with the 2-degree Field (2dF) instrument on the Anglo-Australian Telescope. The quasar candidates were selected to have an extinctioncorrected magnitude $18<g<21.85$ mag. The survey covers a total area of $191.9 \mathrm{deg}^{2}$ in two regions along the celestial equator in the North and South Galactic caps. The $2 \mathrm{dF}$ observations contain new spectra of 16326 objects, of which 8764 are QSOs. A total of 7623 of these are newly discovered, with the remainder previously identified by the 2QZ (Croom et al. 2004) and SDSS (Schneider et al. 2007) surveys. Absolute magnitudes were normalize to $z=2$, rather than the traditional $z=0$, as this minimizes the extrapolation required for the bulk of the objects. We also K-correct to a continuum magnitude, removing the contribution from emission lines. Finally, we make a statistical correction for the host-galaxy contribution, based on the simulations of C09a.

\subsection{The Quasar Luminosity Function}

In order to accurately constrain the luminosity function at both the bright and faint ends, we combine the 2SLAQ sample with the SDSS luminosity function sample, as published by Richards et al. (2006). This gives us an unprecedented combination of precision and dynamic range. The binned luminosity function is calculated using the model-weighted estimator suggested by Miyaji, Hasinger, \& Schmidt (2001). The modelweighted estimator uses the best-fit model to the unbinned LF data (see below) to correct for the variation of the LF within a bin, which is particularly critical at the steep, bright end of the QSO LF. This estimator gives the binned LF as

$$
\Phi\left(M_{g, i}, z_{i}\right)=\Phi\left(M_{g, i}, z_{i}\right)^{\text {model }} \frac{N_{i}^{\text {obs }}}{N_{i}^{\text {model }}},
$$




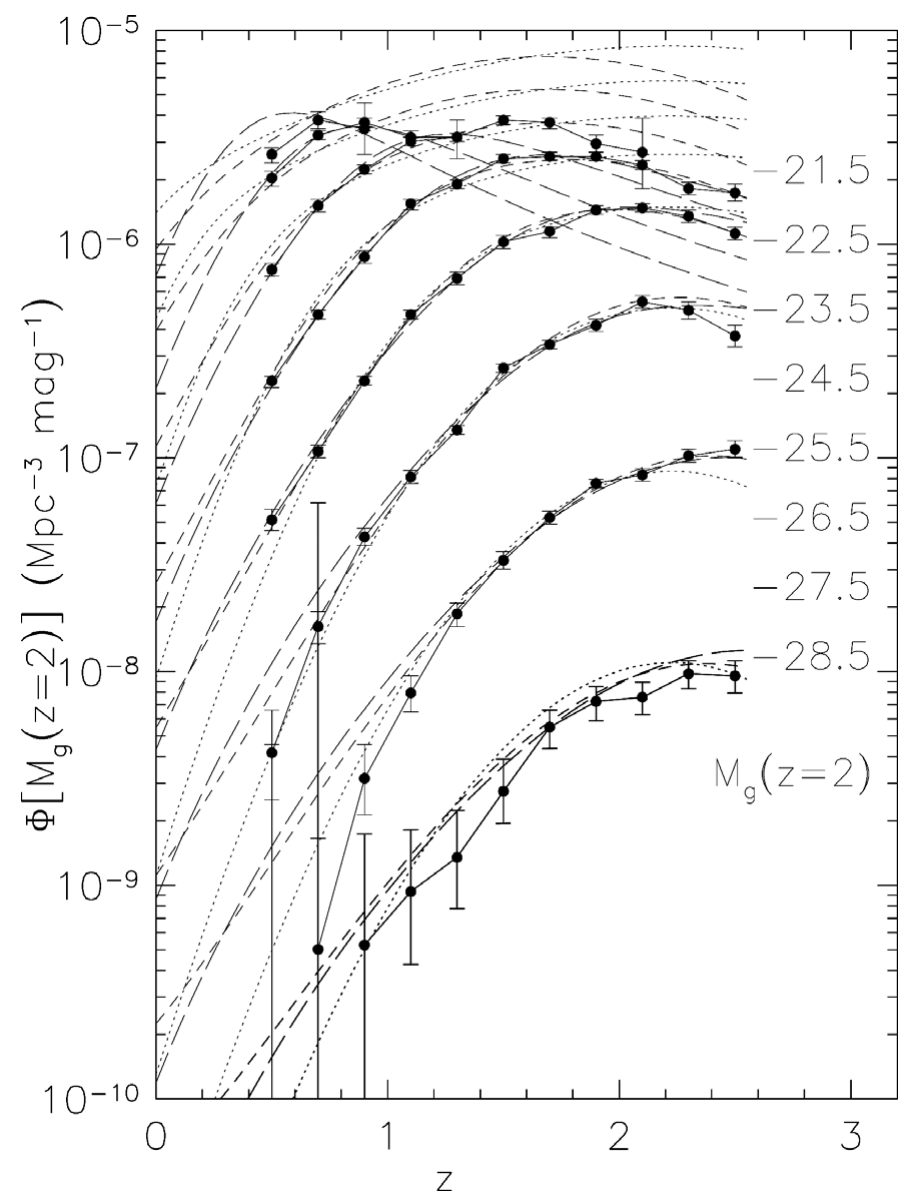

Figure 1. The combined 2SLAQ and SDSS luminosity function plotted as a function of redshift for different $M_{g}$ intervals (from Croom et al. 2009b). The brightest intervals are at the bottom of the plot and the faintest at the top. Each $M_{g}$ interval is connected by a solid line apart from points which are upper limits (e.g., $M_{g}=-27.5 \mathrm{mag}, z=0.5$ ). We compare the measured LF to the best-fit PLE model (dotted lines), smooth LDDE model (long dashed lines) and LEDE model (short dashed lines).

where $M_{g, i}$ and $z_{i}$ are the absolute magnitude and redshift at the center of the $i^{\text {th }}$ bin, $\Phi\left(M_{g, i}, z_{i}\right)^{\text {model }}$ is the best-fit model evaluated at $M_{g, i}$ and $z_{i}$, and $N_{i}^{\text {obs }}$ is the number of observed QSOs in the bin and $N_{i}^{\text {model }}$ the number estimated from the model (accounting for completeness corrections).

In Figure 1, we plot the binned 2SLAQ+SDSS $g$-band luminosity function, as a spacedensity vs. redshift for different absolute magnitude intervals. At bright absolute magnitudes $\left(M_{g} \lesssim-27 \mathrm{mag}\right)$, the space density of QSOs is monotonically increasing up to $z \sim 2.5$. However, the space density of fainter QSOs peaks at lower redshift, e.g., $z \sim 1.6$ for $M_{g}=-23.5 \mathrm{mag}$. This is in disagreement with pure luminosity evolution (PLE) models, in which the space density of QSOs peaks at the same redshift at every luminosity. This is the same trend that has been seen in previous X-ray selected samples of AGN and has been called "AGN downsizing" (e.g., Barger et al. 2005; Hasinger et al. 2005). This downsizing was first seen in X-ray samples because they were the first to have the dynamic range to allow it to be measured. 


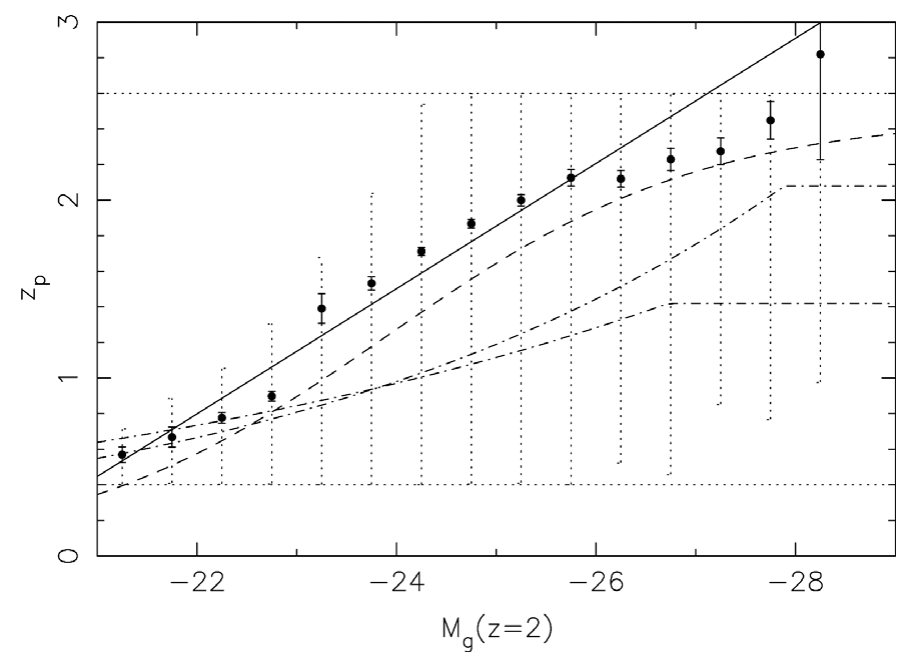

Figure 2. The redshift $z_{\mathrm{p}}$ at which the space density of QSOs peaks as a function of $M_{g}$. This is determined by fitting a quadratic evolution model to our data in narrow slices of full width $\Delta M_{g}=0.5 \mathrm{mag}$. The horizontal dotted lines indicate the nominal redshift range that was fitted over $(z=0.4-2.6)$, while the vertical dotted lines show the actual redshift range for each $M_{g}$ bin. The solid line shows the best linear fit and the dashed line is the best-fit $z_{\mathrm{c}}\left(M_{g}\right)$ using the LDDE model. The dot-dashed lines show the evolution of $z_{\mathrm{c}}$ found by Hasinger et al. (2005; bottom dot-dashed line) and Bongiorno et al. (2007; top dot-dashed line) when fitting a piecewise form of the LDDE model to X-ray and optical data respectively.

The luminosity function we measure is in good agreement with previous, much smaller surveys of faint optical quasars (e.g., Jiang et al. 2006; Wolf et al. 2003; Bongiorno et al. 2007). When we compare our optical luminosity function to the soft X-ray luminosity function of type 1 AGN measured by Hasinger et al. (2005), we find good agreement near $M^{*}$. However, the measured bright-end slope is significantly steeper in the 2SLAQ+SDSS data set than the X-ray data. A natural solution to this is provided by the recent work of Mahony et al. (2009). They find that the fraction of AGN in the ROSAT All-Sky Survey Bright Source Catalogue (BSC) that are detected at radio wavelengths by the NRAO VLA Sky Survey (Condon et al. 1998) and the Sydney University Molonglo Sky Survey (Mauch et al. 2003) increases with increasing redshift. At $z>1$, almost all QSOs in the $\mathrm{BSC}$ are detected in these radio surveys. In fact, the median $1.4 \mathrm{GHz}$ radio flux at $z>1$ is $\sim 1 \mathrm{Jy}$, compared to only $29 \mathrm{mJy}$ for the full sample. This strongly suggests that the brightest high-redshift X-ray sources are only included in a flux-limited sample because their X-ray flux is boosted by a jet component. If this is the case, the bright end of the luminosity function will be boosted in X-ray samples, explaining the difference when compared to optical data.

We fit model luminosity functions to the unbinned data, starting with PLE (see C09b). The PLE model (dotted line in Figure 1) provides a poor fit to the data, as expected given the apparent downsizing. A smoothly varying luminosity-dependent density evolution (LDDE) model (long dashed lines in Figure 1; C09b, Hasinger et al. 2005) is a much improved fit, but we find a best agreement with a model which uses the same basic functional form at the PLE model, but adds a redshift dependence of $\Phi^{*}$ and the bright-end slope (luminosity evolution + density evolution; LEDE, short dashed line in Figure 1). The final fitted parameters are given by Croom et al. (2009b).

To more fully characterize the downsizing of the quasar population, we fit simple luminosity function models to our data in narrow absolute magnitude intervals. We 
assume a single power law in luminosity with quadratic density evolution,

$$
\Phi\left(M_{g}, z\right)=\frac{\Phi^{*} 10^{\left(A z\left(1-0.5 z / z_{\mathrm{p}}\right)\right)}}{10^{0.4(\alpha+1) M_{g}}},
$$

where we fit for $\alpha, A$ and $z_{\mathrm{p}}$. The parameter $z_{\mathrm{p}}$ gives the redshift at which the space density peaks and this is plotted in Figure 2. The best-fit linear relation is found to be $z_{\mathrm{p}}=(-6.94 \pm 0.16)+(-0.352 \pm 0.007) M_{g}$ and a Spearman rank correlation test finds that $z_{\mathrm{p}}$ and $M_{g}$ are correlated at $99.98 \%$ significance. This is the best evidence yet for AGN downsizing (from any data set, including X-ray surveys).

\section{The Quasar Line-Width Distribution}

The broad lines in quasars can, in principle, allow us to estimate black hole masses. This is under the assumption that the gas in the broad-line region is largely in virial equilibrium, orbiting around the black hole. Using this virial assumption, many authors have calculated the distribution of black hole masses for large samples of quasars, such as the SDSS. The virial method makes use of the radius-luminosity relation derived from reverberation mapping, so that the only observables required are velocity width and luminosity. Thus we have

$$
M_{\mathrm{BH}}=A \times L_{\lambda}^{\alpha} \times \sigma_{\mathrm{BLR}}^{2},
$$

where $A$ is a normalizing constant, $L_{\lambda}$ is the monochromatic luminosity at some wavelength $\lambda$ near the emission line, $\alpha$ is $\sim 0.5$ and $\sigma_{\mathrm{BLR}}$ is the width of the emission line. Given that line width and luminosity are the two basic observables, we felt it was wise to consider the properties of the line-width distribution as a function of luminosity, rather than look at correlations involving black hole mass, which inevitably show correlations with luminosity, by construction.

The basic steps in the process which define $L_{\lambda}$ and $\sigma_{\mathrm{BLR}}$ are as follows:

1. Black hole mass $\left(M_{\mathrm{BH}}\right)$ and accretion rate $\left(L / L_{\mathrm{Edd}}\right)$ give us bolometric luminosity, $L_{\mathrm{Bol}}$.

2. $L_{\mathrm{Bol}}$ combined with bolometric corrections and photometric errors give us $L_{\lambda}$, the first observable.

3. $L_{\mathrm{Bol}}$ (or possibly $L_{\mathrm{UV}}$ ) defines the radius of the broad-line region $R_{\mathrm{BLR}}$.

4. $M_{\mathrm{BH}}$ and $R_{\mathrm{BLR}}$, together with the assumption of Keplerian orbits, gives us the velocity of the broad line gas, $V_{\mathrm{BLR}}$.

5. $V_{\mathrm{BLR}}$ together with non-virial motion, orientation effects, and measurement errors give us $\sigma_{\mathrm{BLR}}$, the second observable.

An important point to note is that, apart from uncertainty in the bolometric correction and photometric errors, scatter or errors in each of these steps will increase the scatter in $\sigma_{\mathrm{BLR}}$ at a given luminosity.

We take quasar spectra from the SDSS, 2QZ, and 2SLAQ surveys which contain the $\mathrm{Mg}$ II emission line, as this line allows us to span a wide range in luminosity and redshift. We use the inter-percentile velocity width (IPV; Whittle 1985) as an estimate of line width. The main reason for this is that the correlated errors generated by continuum subtraction can be more transparently accounted for in this approach.

The full distribution in the $L-\sigma_{\mathrm{BLR}}$ plane is shown in Figure 3 . The most obvious feature is a narrowing of the distribution of line-width for the brightest quasars. If we assume a virial relation, in this case using the McLure \& Dunlop (2004) calibration, then we can plot lines of constant black hole mass and Eddington ratio $\left(L / L_{\mathrm{Edd}}\right)$. It 


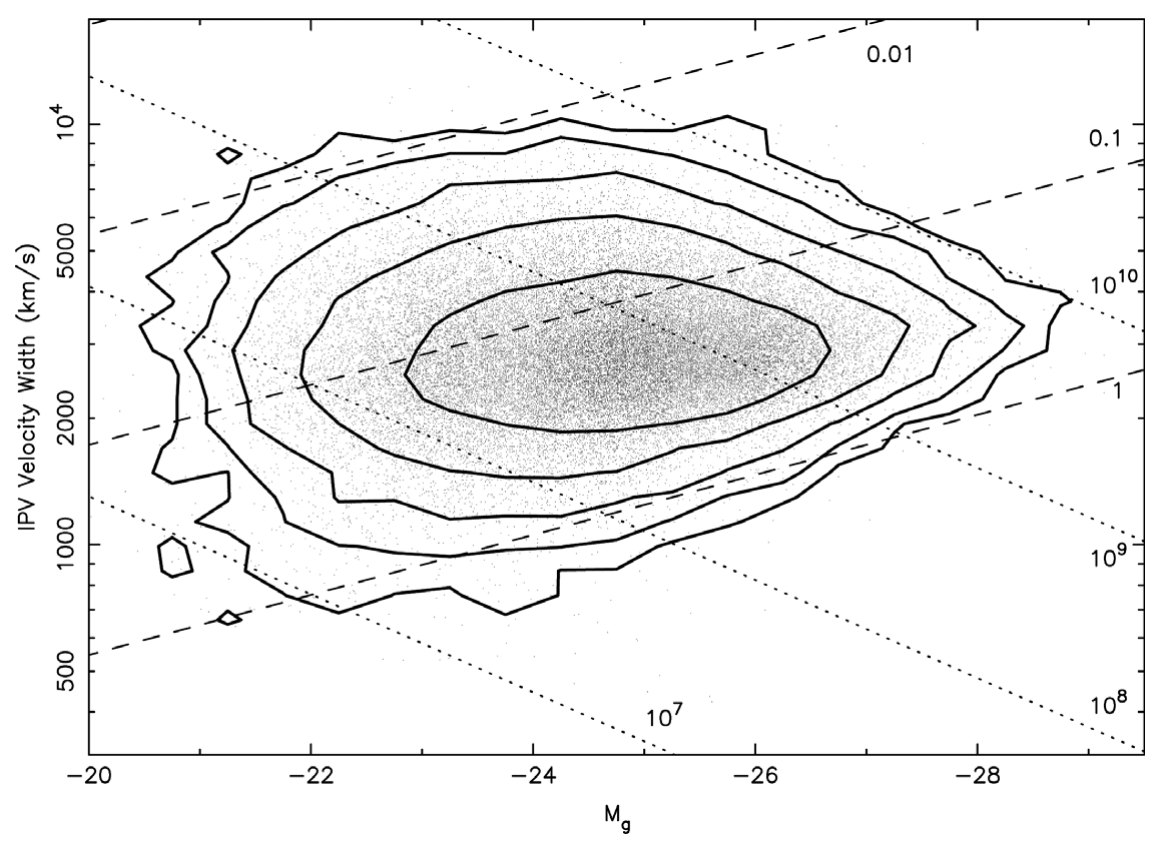

Figure 3. Absolute $g$-band magnitude vs. our measured IPV velocity width for the $\mathrm{Mg}$ II line. Over-plotted are lines of constant black hole mass (dotted) and Eddington ratio $\left(L / L_{\mathrm{Edd}}\right.$; dashed). The contours are evenly spaced in $\log$ (density).

appears that the distribution of line widths is constrained to be at $L / L_{\text {Edd }} \lesssim 1$ and $M_{\mathrm{BH}} \lesssim 10^{10} M_{\odot}$. This constraint is most evident for high-luminosity quasars.

To investigate this further, we measure the dispersion of velocity widths as a function of luminosity (and also redshift). We use an inter-quartile width to define the width of the distribution, as this is less sensitive to outliers than the standard RMS. We subtract off the median measurement error in quadrature to correct to a true scatter. The results are plotted for the three samples separately (and combined) in Figure 4. The first point to note is that the correction for measurement errors (open vs. filled points) is small, and does not significantly change our conclusions. All three data sets are consistent, but the SDSS extends to the highest luminosity. There is a clear correlation between dispersion and luminosity, with the faintest objects having twice the dispersion in log (IPV) as the brightest quasars. Such a relation is in good agreement with the expectation from theoretical models (e.g., Hopkins et al. 2005). In this case, the scatter relates to the range of black hole masses at a given luminosity (i.e., the range of $L / L_{\mathrm{Edd}}$ ). While highluminosity quasars will always be high-mass black holes accreting efficiently, at lower luminosity there can be a mix of high-mass black holes with low accretion rates and low-mass black holes with high accretion rates.

Another striking feature of Figure 4 is the remarkably low scatter seen in the line widths of the highest-luminosity quasars. This is $\sim 0.08$ dex at $M_{g}=-27$ mag, which would correspond to a dispersion of 0.16 dex in black hole mass. Thus, all the terms we discuss above, including scatter in $M_{\mathrm{BH}}$ vs. $\left(L / L_{\mathrm{Edd}}\right)$, scatter in $L_{\mathrm{Bol}}$ vs. $R_{\mathrm{BLR}}$ and orientation effects can only have very small amounts of scatter. Scatter in the luminosityradius relation has been investigated by Kaspi et al. (2005) and Bentz et al. (2006), the latter finding a scatter of $\sim 30 \%$. This is only marginally consistent with the low scatter in the distribution of line widths. However, at high luminosities, where our measurements 


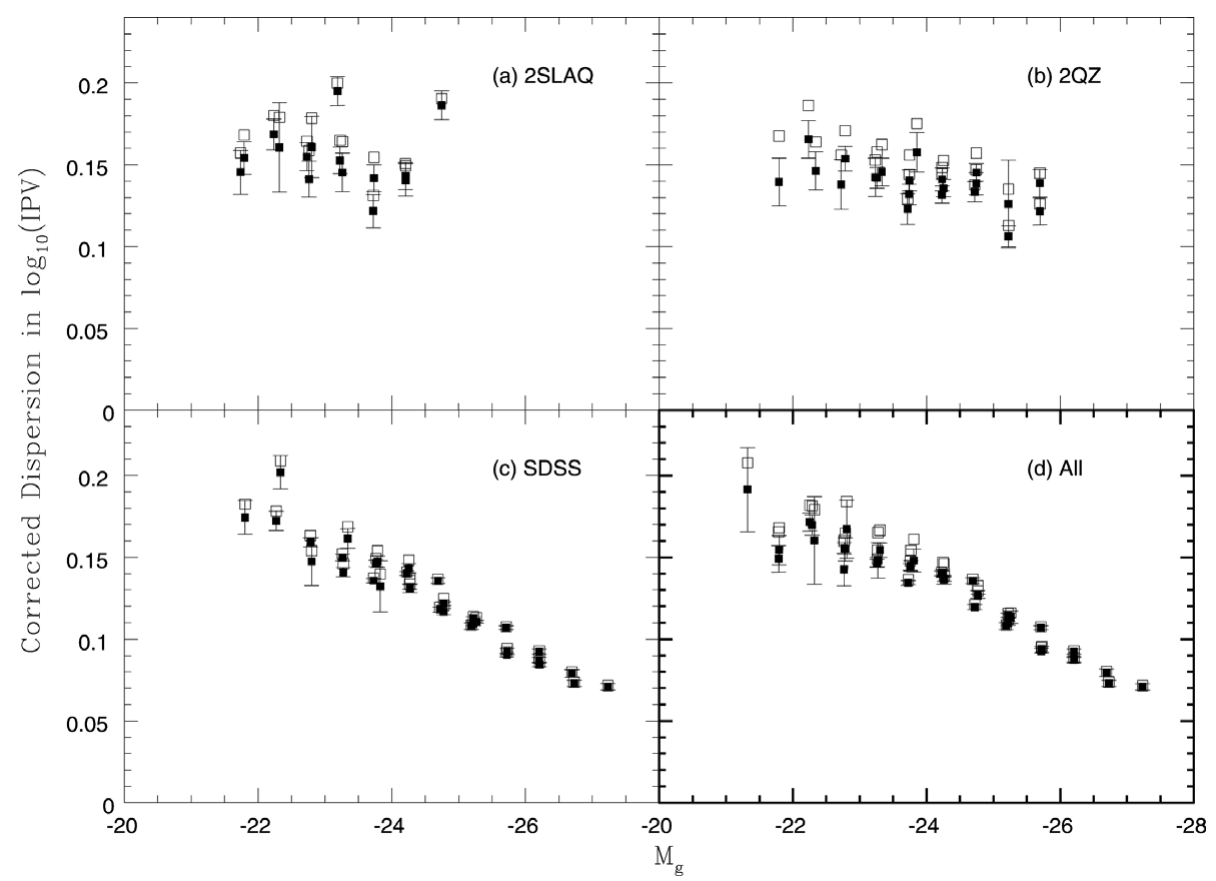

Figure 4. Dispersion in $\log (\mathrm{IPV})$ width as a function of absolute magnitude for (a) $2 \mathrm{SLAQ}$, (b) 2QZ, and (c) SDSS. The combined sample is shown in (d). The results from all redshift bins are plotted on top of each other, as there is no measurable redshift dependence. Open symbols show the dispersion in line-width prior to correction for measurement errors, the filled points (with error bars) are after correction for measurement errors.

are made, the luminosity-radius relation could have considerably less scatter than found by these authors.

The small measured dispersion is line-widths has several implications for virial black hole mass estimates. Possibly the most crucial is that the quoted uncertainty in virial estimators (e.g., Vestergaard \& Peterson 2006) is approximately a factor of 2 greater (in $\log M_{\mathrm{BH}}$ ) than the scatter due to the range of line widths. There is then an argument for simply saying that $M_{\mathrm{BH}} \propto L_{\lambda}^{\alpha}$, and ignoring the line width when estimating mass (at least for the bulk of the population). A second issue to consider is whether the small scatter in line widths is pointing to a fundamental failure of one or more of the assumptions in the virial method. For example, if line width was directly connected to luminosity, and not the black hole mass, a smaller scatter would be natural. However, there is reasonable evidence that the broad-line region is at least partially virialized (e.g., Kollatschny \& Dietrich 1996; Onken \& Peterson 2002).

\section{Summary}

The latest generation of large spectroscopic surveys has enabled major progress to be made in quantifying the demographics of the quasar population. The evolution of quasar luminosity function is now well characterized, and downsizing is apparent in both optical and X-ray samples. Transforming from observed variables (e.g., luminosity and line width) to the variables which drive the physics (e.g., black hole mass and accretion rate) is still a challenge. We demonstrate that the scatter in broad-line velocity width is a function of luminosity, and that for the brightest quasars the scatter is extremely 
small, $\sim 0.08$ dex. While there is reasonable evidence that the broad-line region is at least partially virialized, the low scatter infers that the velocity width gives little information regarding black hole mass.

\section{Acknowledgments}

SMC acknowledges the support of an Australian Research Council QEII Fellowship and a JG Russell Award from the Australian Academy of Science. We warmly thank the organizers of IAU Symposium 276 for an interesting and stimulating meeting.

\section{References}

Alexander, D. M., et al. 2003, AJ, 126, 539

Barger, A. J., et al. 2005, AJ, 129, 578

Bentz, M. C., et al. 2006, ApJ, 644, 133

Bongiorno, A., et al. 2007, A\&广A, 472, 443

Condon, J. J., Cotton, W. D., Greisen, E. W., Yin, Q. F., Perley, R. A., Taylor, G. B., \& Broderick, J. J. 1998, AJ, 115, 1693

Cowie, L. L., Songaila, A., Hu, E. M., \& Cohen, J. G. 1996, AJ, 112, 839

Croom, S. M., et al. 2009, MNRAS, 392, 19 (C09a)

Croom, S. M., et al. 2009, MNRAS, 399, 1755 (C09b)

Croom, S. M., Smith, R. J., Boyle, B. J., Shanks, T., Miller, L., Outram, P. J., \& Loaring, N. S. 2004, MNRAS, 349, 1397

Fine, S., et al. 2008, MNRAS, 390, 1413

Hasinger, G., et al. 2001, A\&\&A, 365, L45

Hasinger, G., Miyaji, T., \& Schmidt, M. 2005, A\& $A, 441,417$

Hopkins, P. F., Hernquist, L., Cox, T. J., Di Matteo, T., Robertson, B., \& Springel, V. 2005b, ApJ, 630, 716

Jiang, L., et al. 2006, AJ, 131, 2788

Juneau, S., et al. 2005, ApJ, 619, 135

Kaspi, S., et al. 2005, ApJ, 629, 61

Kollatschny, W. \& Dietrich, M. 1996, A\&A, 314, 43

Kormendy, J. \& Richstone, D. 1995, ARAA, 33, 581

Mahony, E. K., Croom, S. M., Boyle, B. J., Edge, A. C., Mauch, T., \& Sadler, E. M. 2010, MNRAS, 401, 1151

Mauch, T., et al. 2003, MNRAS, 342, 1117

McLure, R. J. \& Dunlop, J. S. 2004, MNRAS, 352, 1390

McLure, R. J. \& Jarvis, M. J. 2002, MNRAS, 337, 109

Miyaji, T., Hasinger, G., \& Schmidt, M. 2001, A\&A, 369, 49

Onken, C. A. \& Peterson, B. M. 2002, ApJ, 572, 746

Richards, G. T., et al. 2006, AJ, 131, 2766

Schlegel, D. J., Finkbeiner, D. P., \& Davis, M. 1998, ApJ, 500, 525

Schneider, D. P., et al. 2007, AJ, 134, 102

Tremaine, S., et al. 2002, ApJ, 574, 740

Ueda, Y., Akiyama, M., Ohta, K., \& Miyaji, T. 2003, ApJ, 598, 886

Vestergaard, M. \& Peterson, B. M. 2006, ApJ, 641, 689

Wandel, A., Peterson, B. M., \& Malkan, M. A. 1999, ApJ, 526, 579

Whittle, M. 1985, MNRAS, 213, 1

Wolf, C., Wisotzki, L., Borch, A., Dye, S., Kleinheinrich, M., \& Meisenheimer, K. 2003, A\& A, 408, 499 